\title{
Wave Packet Echoes in the Motion of Trapped Atoms
}

\author{
F. B. J. Buchkremer, R. Dumke, H. Levsen, G. Birkl, and W. Ertmer \\ Institut für Quantenoptik, Universität Hannover, Welfengarten 1, D-30167 Hannover, Germany
}

(Received 1 March 2000)

\begin{abstract}
We experimentally demonstrate and systematically study the stimulated revival (echo) of motional wave packet oscillations. For this purpose, we prepare wave packets in an optical lattice by nonadiabatically shifting the potential and stimulate their reoccurrence by a second shift after a variable time delay. This technique, analogous to spin echoes, enables one even in the presence of strong dephasing to determine the coherence time of the wave packets. We find that for strongly bound atoms it is comparable to the cooling time and much longer than the inverse of the photon scattering rate.
\end{abstract}

PACS numbers: $32.80 . P \mathrm{j}, 42.50 . \mathrm{Vk}$

The process of decoherence, i.e., the collapse of superposition states due to the dissipative interaction with their environment, is one of the basic concepts for our understanding of the connection between classical and quantum physics. In order to study the effect of decoherence unambiguously, one has to be able to distinguish it from other, nondissipative effects. The macroscopic (i.e., ensemble- or time-averaged) response of a quantum system prepared in a superposition state typically decays not only due to the loss of coherence (homogeneous decay) but also due to dephasing resulting from local variations in the evolution of the quantum system (inhomogeneous decay). In many cases decoherence cannot be studied directly because the inhomogeneous decay is by far the dominating process.

This limitation has been overcome in a famous series of experiments by introducing the techniques of spin echo for nuclear magnetic resonance and photon echo for optical resonance $[1-3]$. These techniques are based on the observation that inhomogeneous decay due to dephasing is a reversible process. Thus, by appropriately modifying superposition states at a time $\Delta t$ after their preparation, the dephasing can be partially or fully reversed and a stimulated macroscopic response (echo) is induced at $2 \Delta t$. This effect enables one to measure the coherence time even in the presence of strong dephasing. We have, for the first time, applied this method to the investigation of the decoherence of motional wave packets of trapped atoms (Fig. 1). The method can be used independent of the specific experimental realization of the confining potential (e.g., a single dipole potential, periodic dipole potentials, magnetic trapping potentials, inhomogeneous arrays of atom traps, etc.).

The specific system investigated here consists of motional wave packets of neutral atoms in a one-dimensional optical lattice. Optical lattices are periodic dipole potentials for atoms created by the interference of multiple laser beams [4]. Atoms can be trapped and cooled at the potential minima (mean position spread $z_{\mathrm{rms}}=\lambda / 18$ [5]). In optical lattices symmetrically and asymmetrically oscillating motional wave packets can be induced by nonadiabatically changing the lattice potential [6-11].
In dipole potentials, the macroscopic oscillation signal decays because of decoherence due to the spontaneous scattering of photons and because of dephasing due to the anharmonicity of the potential wells and spatial variations of the potential depth. Typically, the effect of dephasing is dominating decoherence [6-13], so that a direct determination of the coherence time is not possible. Here, we show how these limitations can be overcome: For the case of symmetrical oscillations, Bulatov et al. [14] recently proposed and numerically simulated an echo mechanism to reverse the effect of dephasing and stimulate the revival of the wave packet oscillations by means of two successive nonadiabatic changes in the depth of the lattice potential. With our work we extend their proposal to the case of asymmetrical wave packet oscillations, present the first experimental observation of the echo effect, and apply it to determine the coherence time of the motional wave packets.

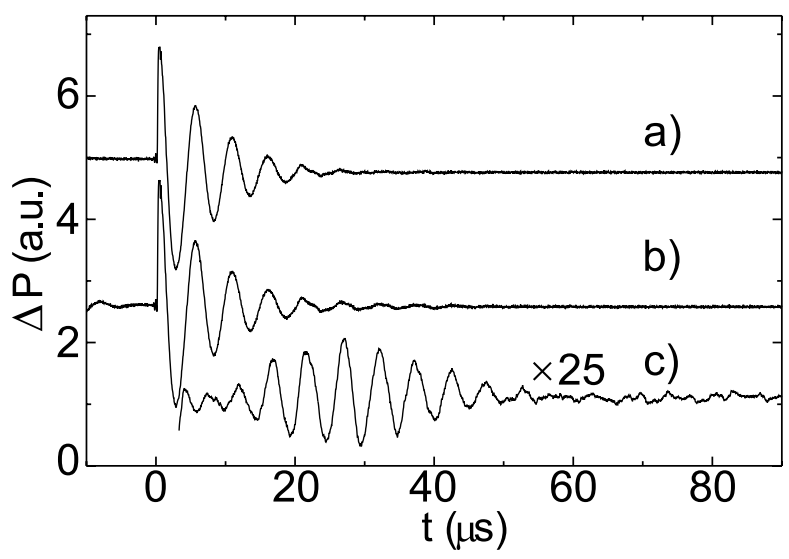

FIG. 1. Wave packet oscillations without (curve $a$ ) and with (curves $b, c)$ stimulated revival (echo) $\left(U_{0}=831 E_{R}\right.$, $\delta=-7.8 \Gamma, d z=0.10 \lambda)$. Depicted are the oscillations after the second shift (at $t=0$ ) for time delays between the two shifts of $\Delta t=108 \mu \mathrm{s}$ ( $a$, reference curve, complete decoherence before second shift) and $\Delta t=32 \mu \mathrm{s}$ ( $b$, signal curve), showing additional oscillations at about $t=32 \mu \mathrm{s}$. The difference between curves $b$ and $a$ ( $c$, echo curve) shows the net effect of the wave packet echo. 
In our experiment, we chirp-slow rubidium atoms $\left({ }^{85} \mathrm{Rb}\right)$ from a thermal beam and trap and cool them in a magneto-optical trap (MOT), giving a sample of approximately $10^{7}$ atoms with a central density of $10^{9}$ atoms $/ \mathrm{cm}^{3}$. After the loading phase, the magnetic field of the MOT is switched off and the intensity of the trapping beams is reduced to achieve optimal cooling in a three-dimensional molasses. The MOT-laser beams are then turned off and two lattice beams are switched on, forming a onedimensional lin $\perp$ lin optical lattice [4]. The lattice beams have intensities up to $I=60 \mathrm{~mW} / \mathrm{cm}^{2}$, and detunings $\delta$ of 2-10 natural linewidths $(\Gamma / 2 \pi=5.89 \mathrm{MHz})$ below the $5 S_{1 / 2}(F=3) \rightarrow 5 P_{3 / 2}\left(F^{\prime}=4\right)$ transition at $\lambda=780 \mathrm{~nm}$. The beam waist of about $2.75 \mathrm{~mm}\left(1 / e^{2}\right.$ radius) is large compared to the $1 / e^{2}$ radius of the atomic cloud of $1.52 \mathrm{~mm}$.

After an initial cooling phase of 1 to $2 \mathrm{~ms}$ in the lattice, which localizes the atoms at the center of the potential wells, we nonadiabatically change the relative phase between the two lattice beams with an electro-optic phase shifter [11] $(1 / e$ switching time of $0.4 \mu \mathrm{s})$. This causes a translation of the lattice by a controllable amount $d z(0<$ $d z<\lambda / 4)$ and induces asymmetrical coherent-state-like motional wave packets. We observe the wave packet oscillations by measuring the photon redistribution-induced power difference $\Delta P(t)$ between the two lattice beams $[11,15]$. No repumping light is present during the wave packet evolution. Figure 1(a) shows a typical example of a wave packet oscillation. Clearly visible are about five oscillations with a period of $(5.2 \pm 0.1) \mu \mathrm{s}$. The oscillation signal is damped with a decay time of $\tau_{1}=7 \pm 1 \mu \mathrm{s}$ (exponential fit).

Figure 2 shows the decay time $\tau_{1}$ (relative uncertainty $\leq 20 \%$ ) of asymmetrical wave packet oscillations as a func-

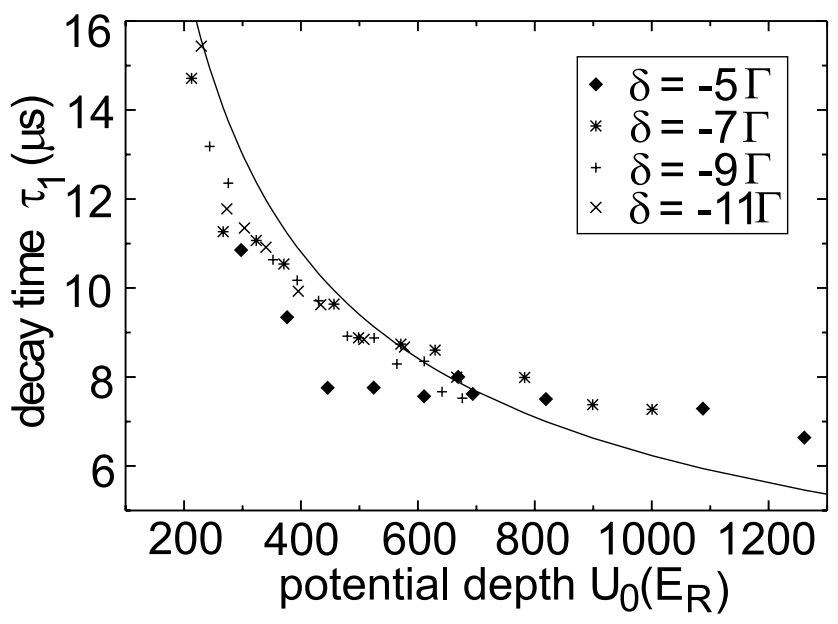

FIG. 2. Decay times $\tau_{1}$ of asymmetrical wave packet oscillations as a function of the potential depth $U_{0}$ for the indicated values of the detuning $\delta$. The solid line shows a calculation of the decay time for anharmonicity-induced dephasing as the decay mechanism. tion of the potential depth $U_{0}$ for various detunings $\delta$. $U_{0}$ is calculated from the measured oscillation frequency $\omega_{\text {osc }}$ according to $U_{0} / E_{R}=\left[1 /(0.86)^{2}\right]\left(\omega_{\text {osc }} / 2 \omega_{R}\right)^{2}$, taking into account the anharmonicity of the potential wells $[5,9]$. The data show that the decay is not due to decoherence caused by spontaneous scattering, for in that case the decay time $\tau_{1}$ should be proportional to the inverse of the photon scattering rate, $\tau_{\mathrm{sc}}=1 / \Gamma^{\prime}=1 /\left[U_{0} \Gamma /(\hbar|\delta|)\right]$. For different detunings this should result in different decay times $\tau_{1}$ for the same $U_{0}$, which is not observed for $|\delta| \geq 7 \Gamma$. For a decay caused by anharmonicity, on the other hand, the decay time should be proportional to the inverse of the mean spread $\Delta \omega_{\text {osc }}$ of the occurring oscillation frequencies, which can be approximated by the mean shift of the oscillation frequencies from the harmonic frequency minus the common shift of $\omega_{R}$, i.e., $\Delta \omega_{\text {osc }} \approx 0.14 \omega_{\text {osc }}-\omega_{R}[5,9]$. The line in Fig. 2 is a plot of $1 / \Delta \omega_{\text {osc }}$ as a function of $U_{0}$. Within the $20 \%$ uncertainty of our data the decay times $\tau_{1}$ are in agreement with this calculation. Thus, the decay of the wave packet oscillations is dominated by dephasing [16].

The investigation of decoherence, on the other hand, becomes possible by nonadiabatically shifting the lattice back to its initial position after a variable delay $\Delta t$. This second translation leads to a stimulated revival (echo) of the oscillations with a maximum amplitude at about $2 \Delta t$ [14] if the coherence of the wave packets induced by the first shift still persists - at least partially — at $2 \Delta t$.

Figure 1 shows the first experimental demonstration of this echo effect [17]. Figure 1(a) serves as a reference curve showing wave packet oscillations for atoms that move fully incoherently at the time of the shift. For the purpose of reducing systematic uncertainties, this curve also has been recorded after two translations of the lattice. However, here the long delay time $\Delta t=108 \mu \mathrm{s}$ guarantees a complete loss of coherence before the second shift. Figure 1(b) depicts the wave packet oscillations after the second shift with $\Delta t=32 \mu \mathrm{s}$. The curve shows additional oscillations at about $t=32 \mu \mathrm{s}$. Figure 1(c) presents the magnified difference of curves $(b)$ and $(a)$. Clearly visible is the recurrence of wave packet oscillation, i.e., echo, centered around a time close to $t=32 \mu \mathrm{s}$. This time corresponds to a total time of $t_{\text {total }}=32 \mu \mathrm{s}+\Delta t=$ $64 \mu \mathrm{s}=2 \Delta t$ after the first shift, as predicted.

In order to gain a qualitative and quantitative understanding of the echo mechanism, we have performed a full quantum Monte Carlo wave-function simulation (QMCWF) [18] of the echo experiment, taking into account the full coherent and dissipative dynamics as well as all internal ground states while adiabatically eliminating the excited states. Figure 3 shows the result for the experimental conditions of Fig. 1. In Fig. 3(a) the spontaneous scattering rate is reduced $\left(\Gamma^{\prime} \rightarrow \Gamma^{\prime} / 1000\right)$ in order to emphasize the coherent dynamics which gives rise to the echo mechanism. Clearly visible is the occurrence of the wave packet echo at $t=32 \mu \mathrm{s}$. 


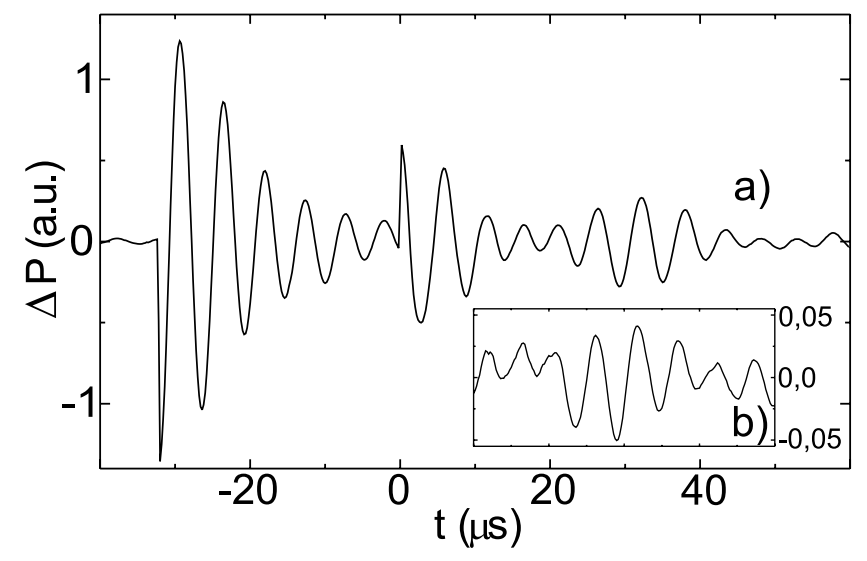

FIG. 3. QMCWF simulations of the echo experiment $\left[I=56.5 \mathrm{~mW} / \mathrm{cm}^{2} \quad\right.$ and $\delta=-7.8 \Gamma \quad\left(\Rightarrow U_{0}=823 E_{R}\right)$, $d z=0.064 \lambda, \Delta t=32 \mu \mathrm{s}$ (potential shifts at $t=-32 \mu \mathrm{s}$ and $t=0)]$. (a) With a reduced rate of spontaneous scattering processes $\left(\Gamma^{\prime} \rightarrow \Gamma^{\prime} / 1000\right)$, a strong wave packet echo is visible. (b) With the regular rate of spontaneous scattering, the echo is still visible but its amplitude is significantly reduced.

The underlying physics of the echo mechanism can be understood in terms of the coherent evolution of the contributions of different eigenstates to the wave packet: The first translation creates a coherent superposition of eigenstates of the translated anharmonic trapping potential which are all in phase (with phase $=0$ per definition). The phases of different eigenstates evolve with different frequencies in time which causes the decay of the oscillation signal. Shifting the lattice back after $\Delta t$ creates a new superposition state. Because of the asymmetry of the translation, eigenstates with phases close to odd multiples of $\pi$ give the strongest and eigenstates with phases close to even multiples of $\pi$ give the weakest contributions to the new superposition state, which leads to new wave packet oscillations which again dephase in time. However, eigenstates that were in phase at $t=\Delta t$ are again in phase at $t=2 \Delta t$, so that the strongest contributions again realign at $t=2 \Delta t$, which results in a partial revival of the oscillations. Thus, the phase-dependent selection of the strength of eigenstate contributions at the second shift leads to the wave packet echo.

Spontaneous scattering leads to an incoherent evolution of the superposition state by randomizing the phases of the eigenstates and thus causes a decrease in the echo amplitude. This is clearly observed in our wave-function simulation for a nonreduced spontaneous scattering rate [Fig. 3(b)]. The wave packet echo is still visible but its amplitude is reduced to a value that is consistent with the one obtained in the experiment (Fig. 1).

In order to experimentally study additional effects of dephasing, we have narrowed the transverse intensity profile of our lattice beams, causing different parts of the atom cloud to experience different potential depths $U_{0}$.

The almost freestanding echo in the signal curve of Fig. 4 experimentally demonstrates, that the method of

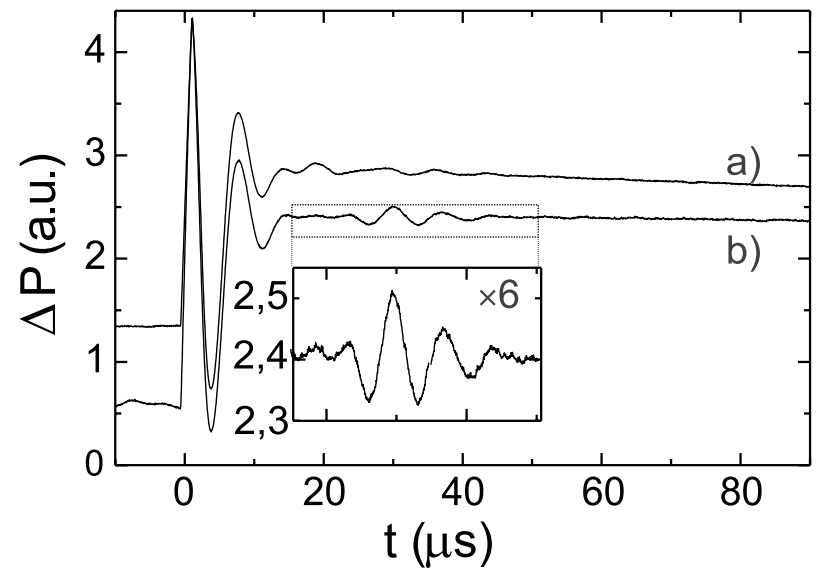

FIG. 4. Wave packet oscillations and echo for increased dephasing $\left(U_{0}=368 E_{R}, \delta=8 \Gamma, d z=0.11 \lambda\right)$. The reference curve $(a, \Delta t=132 \mu \mathrm{s})$ depicts the faster signal decay. The signal curve $(b, \Delta t=30 \mu \mathrm{s})$ shows a freestanding echo.

wave packet echoes can also be applied to other systems, where the spatial variation in the coherent dynamics is the dominating effect for dephasing as, e.g., in anharmonic dipole traps based on focused laser beams, inhomogeneous arrays of atom traps, or magnetic quadrupole traps, etc. (see, e.g., Ref. [13]).

The physics behind the echo effect predicts that the time at which the echo occurs can be varied by changing the time delay $\Delta t$ between lattice shifts. Figure 5 shows echo curves [analogous to Fig. 1(c)] for the indicated values of the time delay $\Delta t$. For each curve, $t=0$ corresponds to the second lattice shift. The total time after the first shift is given by $t_{\text {total }}=t+\Delta t$. Our measurements confirm that the echo moves to larger $t$ with increasing $\Delta t$ and has its maximum value at approximately $t_{\text {total }}=2 \Delta t$ [19].

We can use the echo technique to determine the coherence time $\tau_{2}$ of the atomic motion by measuring the echo amplitude as a function of the echo time $2 \Delta t$. Figure 6(a) shows this for the data of Fig. 5. With increasing $\Delta t$

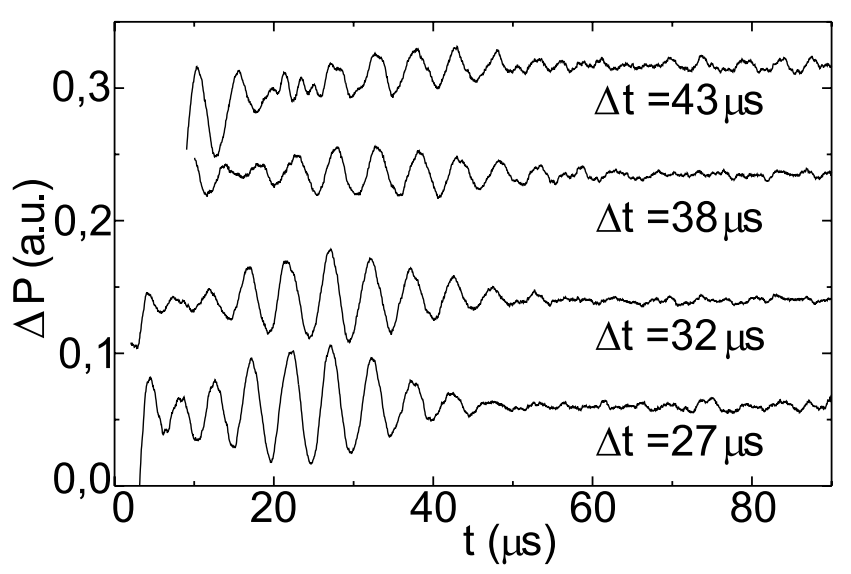

FIG. 5. Increase in occurrence time and decrease in amplitude of the echoes for increasing delay $\Delta t$ between translations of the trapping potential (parameters as in Fig. 1). 


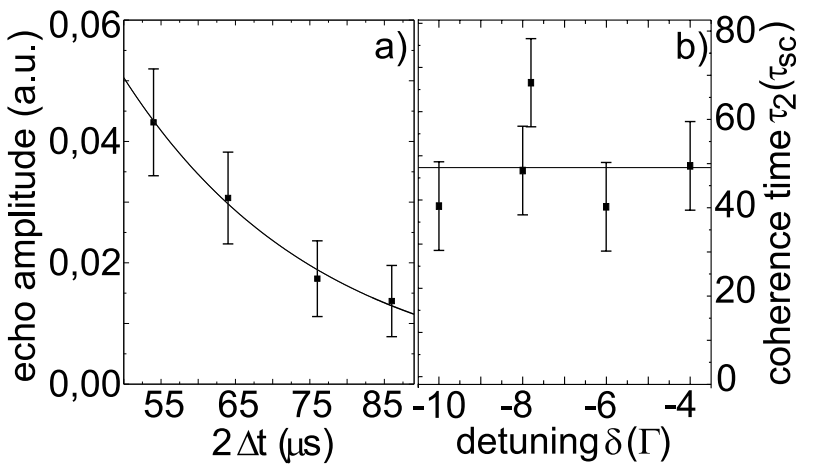

FIG. 6. (a) Dependence of the echo amplitude on the echo time $2 \Delta t$ for the data of Fig. 5. The line is an exponential fit to the data, giving a coherence time of $\tau_{2}=(27 \pm 3) \mu \mathrm{s}$. (b) Coherence time in units of the photon scattering time $\tau_{\mathrm{sc}}=$ $1 / \Gamma^{\prime}$ as a function of the lattice detuning $\delta$.

the echo amplitude decreases exponentially as expected for a coherence loss due to spontaneous scattering processes occurring at a constant rate $\Gamma^{\prime}$. An exponential fit to the data [line in Fig. 6(a)] gives a $1 / e$ decay time of $\tau_{2}=(27 \pm 3) \mu$ s which is significantly longer than the observed dephasing time of $7 \mu \mathrm{s}$. This shows that the coherent motion still persists for times at which the macroscopic oscillation signal has already disappeared.

The echo technique even enables a quantitative determination of the constant of proportionality between the coherence time $\tau_{2}$ and the photon scattering time $\tau_{\mathrm{sc}}=1 / \Gamma^{\prime}$. For the data of Fig. $6(\mathrm{a}), \tau_{\mathrm{sc}}=(0.40 \pm 0.04) \mu \mathrm{s}$ and we find that, during the coherence time, one atom scatters $68 \pm 10$ photons. In a series of measurements similar to Fig. 5 for $-10 \Gamma \leq \delta \leq-4 \Gamma$ we observe coherence times in the range from 40 to 68 scattering times $\tau_{\text {sc }}$ with an average value of $\tau_{2}=(49 \pm 7) \tau_{\mathrm{sc}}$ independent of the detuning $\delta$ [Fig. 6(b)]. This proves that the coherence time $\tau_{2}$ is proportional to the scattering time $\tau_{\mathrm{sc}}$, and that for the decay of motional coherence a large number of photons has to be scattered spontaneously.

The quantitative determination of the coherence time enables us to prove an important prediction given for the dissipative coupling of the motion of atoms in (nearly) harmonic potentials to their environment: It has been pointed out that for (nearly) harmonic oscillators the coherence time $\tau_{2}$ should be twice as long as the damping time of the oscillator's energy, i.e., the cooling time $\tau_{\text {cool }}$ of the atoms [20-22]. In a recent experiment, $\tau_{\text {cool }}$ in onedimensional optical lattices was found to be the time to spontaneously scatter 30 photons: $\tau_{\text {cool }}=30 \tau_{\text {sc }}$ [7]. Our direct measurement of the coherence time $\tau_{2}=(49 \pm$ 7) $\tau_{\mathrm{sc}}$ is the first experimental confirmation of the predicted relation between the coherence time and the cooling time. We expect the relation $\tau_{2}=2 \tau_{\text {cool }}$ to hold universally for (nearly) harmonic trapping potentials in which the motion of atoms is coupled to a dissipative reservoir [20].

In summary, we have experimentally demonstrated and systematically investigated a new echo technique to access the coherent and dissipative dynamics of atomic wave packet oscillations in trapping potentials. With this technique, we have measured the coherence time of wave packet oscillations in strongly confining dipole potentials, in a regime, which was previously inaccessible. We showed that the coherence time is directly connected to the energy dissipation time. We also demonstrated that the echo technique works for atomic motion exhibiting various causes of dephasing, which shows that it can be used to access the external dynamics in a broad range of different atom traps or inhomogeneous arrays of atom traps.

We thank A. Pahl and M. Wilken for their support in the early stages of the project. This work is supported by the SFB 407 of the Deutsche Forschungsgemeinschaft.

[1] E. L. Hahn, Phys. Rev. 80, 580 (1950).

[2] N. A. Kurnit, I. D. Abella, and S. R. Hartmann, Phys. Rev. Lett. 13, 567 (1964).

[3] L. Allen and J.H. Eberly, Optical Resonance and TwoLevel Atoms (Dover, New York, 1987).

[4] P. S. Jessen and I. H. Deutsch, Adv. At. Mol. Opt. Phys. 37, 95 (1996); D. R. Meacher, Contemp. Phys. 39, 329 (1998); L. Guidoni and P. Verkerk, J. Opt. B 1, R23 (1999).

[5] M. Gatzke et al., Phys. Rev. A 55, R3987 (1997).

[6] M. Kozuma et al., Phys. Rev. Lett. 76, 2428 (1996).

[7] G. Raithel et al., Phys. Rev. Lett. 78, 630 (1997).

[8] A. Görlitz et al., Phys. Rev. Lett. 78, 2096 (1997).

[9] G. Raithel et al., Phys. Rev. Lett. 78, 2928 (1997).

[10] P. Rudy, R. Ejnisman, and N. P. Bigelow, Phys. Rev. Lett. 78, 4906 (1997); R. Ejnisman et al., Phys. Rev. A 56, 4331 (1997).

[11] G. Raithel, W. D. Phillips, and S. L. Rolston, Phys. Rev. Lett. 81, 3615 (1998).

[12] O. Morsch, P. H. Jones, and D. R. Meacher, Phys. Rev. A 61, 023410 (2000).

[13] M. Morinaga et al., Phys. Rev. Lett. 83, 4037 (1999).

[14] A. Bulatov et al., Phys. Rev. A 57, 3788 (1998).

[15] The maximum power difference $\Delta P$ is about $1 \%$ of the total lattice power for all data presented here.

[16] The remaining deviations may be due to the additional influence of decoherence and local variations in $U_{0}$.

[17] In order to compensate fluctuations in atom number, the initial amplitudes of all wave packet oscillations are normalized. For clarity some curves are offset vertically.

[18] J. Dalibard, Y. Castin, and K. Mølmer, Phys. Rev. Lett. 68, 580 (1992); P. Marte et al., Phys. Rev. A 47, 1378 (1993); P. Marte et al., Phys. Rev. Lett. 71, 1335 (1993).

[19] The fact that the maxima occur at times slightly earlier than $2 \Delta t$ is caused by decoherence, which decreases the signal strength during the echo, thus shifting the apparent echo maximum to earlier times.

[20] W. D. Phillips and C. I. Westbrook, Phys. Rev. Lett. 78, 2676 (1997).

[21] J. I. Cirac et al., Phys. Rev. A. 48, 2169 (1993).

[22] C. Cohen-Tannoudji, J. Dupont-Roc, and G. Grynberg, Atom-Photon Interactions (Wiley, New York, 1992). 